\title{
Sclerosing cholangitis associated with multifocal fibrosis: a case report
}

\author{
R D Laitt, S G Hubscher, J A Buckels, S Darby, E Elias
}

\begin{abstract}
The association of sclerosing cholangitis, retroperitoneal fibrosis, and Riedel's thyroiditis has been reported twice before, ${ }^{12}$ and on both occasions the authors successfully used steroids to control the fibrotic process. A further case of fibrosis with this triad of organ involvement in which a combination of surgery and steroid treatment has arrested disease progression is described. This suggests an inappropriate immune response in this type of fibrotic overlap syndrome.

(Gut 1992; 33: 1430-1432)
\end{abstract}

Abnormal fibrous tissue overgrowth has long been known to affect a number of widely separate organ systems. The terms Riedel's thyroiditis, sclerosing cholangitis, retroperitoneal fibrosis, mediastinal fibrosis, and pseudotumour of the orbit reflect the multiple areas affected. These fibrotic processes are rare and tend to occur in isolation, although a number of overlap syndromes has been reported with multiple organ involvement. This overlap was first described by Bowlby ${ }^{3}$ in 1885 , in a necropsy report that commented on a hard infiltrative mass affecting the thyroid and other structures in the neck contiguous with similar tissue throughout the mediastinum. Barrett ${ }^{+}$was the first to suggest that these fibrotic conditions were interrelated and probably different manifestations of the same disorder. Patients in whom fibrosis occurred in a combination of sites, particularly the biliary tree and retroperitoneal area, are well documented in a review by Lillemoe et al. ${ }^{5}$ However, the association of fibrous thyroiditis with other sites of fibrosis was not widely noted until 1962 when Hache ${ }^{6}$ reported an association with retroperitoneal fibrosis and Woolner ${ }^{7}$ an association with sclerosing cholangitis. A year later Bartholemew' noted an association of Riedel's thyroiditis, sclerosing cholangitis, and retroperitoneal fibrosis in the same patient. Gleeson $^{2}$ is the only other author to have documented this pattern of organ involvement. Both authors used steroids in an attempt to control the disease process, with some success. We report a further patient with Reidel's thyroiditis, sclerosing cholangitis, and retroperitoneal fibrosis whose condition improved coincident with steroid therapy.

\section{Case report}

A 51 year old woman was admitted to another hospital in February 1988 with a two month history of malaise and swelling in the neck. There was no relevant drug history. On physical examination she was afebrile but looked hypo- thyroid with a large, firm, generalised thyroid swelling. Her blood pressure was normal. Hypothyroidism was confirmed by a serum thyroid stimulating hormone concentration of more than $50 \mathrm{mU} / \mathrm{l}$ (normal range $<6 \mathrm{mU} / \mathrm{l}$ ) and a thyroxine concentration of $29 \mathrm{nmol} / \mathrm{l}(70-140 \mathrm{nmol} / \mathrm{l})$. Thyroid autoantibodies were weakly positive. Thyroxine replacement treatment was begun with some improvement but by May the goitre had increased in size and began to cause local pressure effects.

Thyroidectomy was performed without problem or need for blood transfusion. Notes taken at the time of operation commented on the appearances of 'an anaplastic thyroid carcinoma infiltrating strap muscles anteriorly and carotid sheath posteriorly. Formal resection was not possible.' Histology (Fig 1) showed complete effacement of the normal thyroid architecture and its replacement by dense collagenous and inflamed fibrous tissue. The inflammatory process was seen to extend into adjacent skeletal muscle and was consistent with a diagnosis of Riedel's thyroiditis. She recovered well and was allowed home on thyroxine replacement treatment.

In July 1988 the patient presented again to the same hospital with a three week history of generalised pruritus, painless jaundice, dark urine, and pale stool. Physical examination confirmed jaundice and the presence of hepatomegaly. Results of laboratory studies included bilirubin concentration $156 \mu \mathrm{mol} / \mathrm{l}$ (normal range 3-17 $\mu \mathrm{mol} / \mathrm{l}$ ), alkaline phosphatase 3000 IU/1 (30-300 IU/1), alanine aminotransferase of 179 IU/1 (5-35 IU/1), and aspartate aminotransferase activity $147 \mathrm{IU} / \mathrm{l}(5-35 \mathrm{IU} / \mathrm{l})$.

An endoscopic cholangiogram showed obstruction within the common hepatic duct. She was then transferred to this centre. Examination findings were confirmed as was the

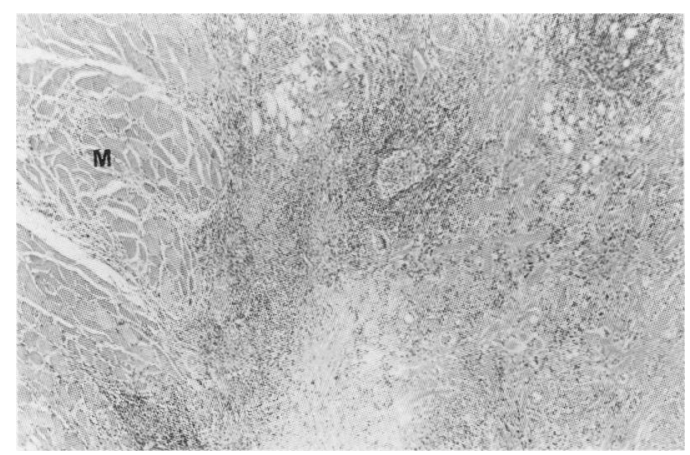

Figure 1: Sections of thyroid mass discovered at neck exploration. No residual thyroid tissue is present. The specimen is composed of fibrous tissue containing a dense infiltrate of chronic inflammatory cells. Extension into skeletal
intecimen in muscle $(M)$ is seen to the left. (Haematoxylin and eosin original magnification $\times 55$. 
Figure 2: Variations in patient's serum bilirubin concentration with time.

Figure 3: Percutaneous transhepatic cholangiogram showing multiple strictures within the intrahepatic

biliary tree. Appearances are consistent with a diagnosis of sclerosing cholangitis. A prosthesis has been introduced into the main duct.

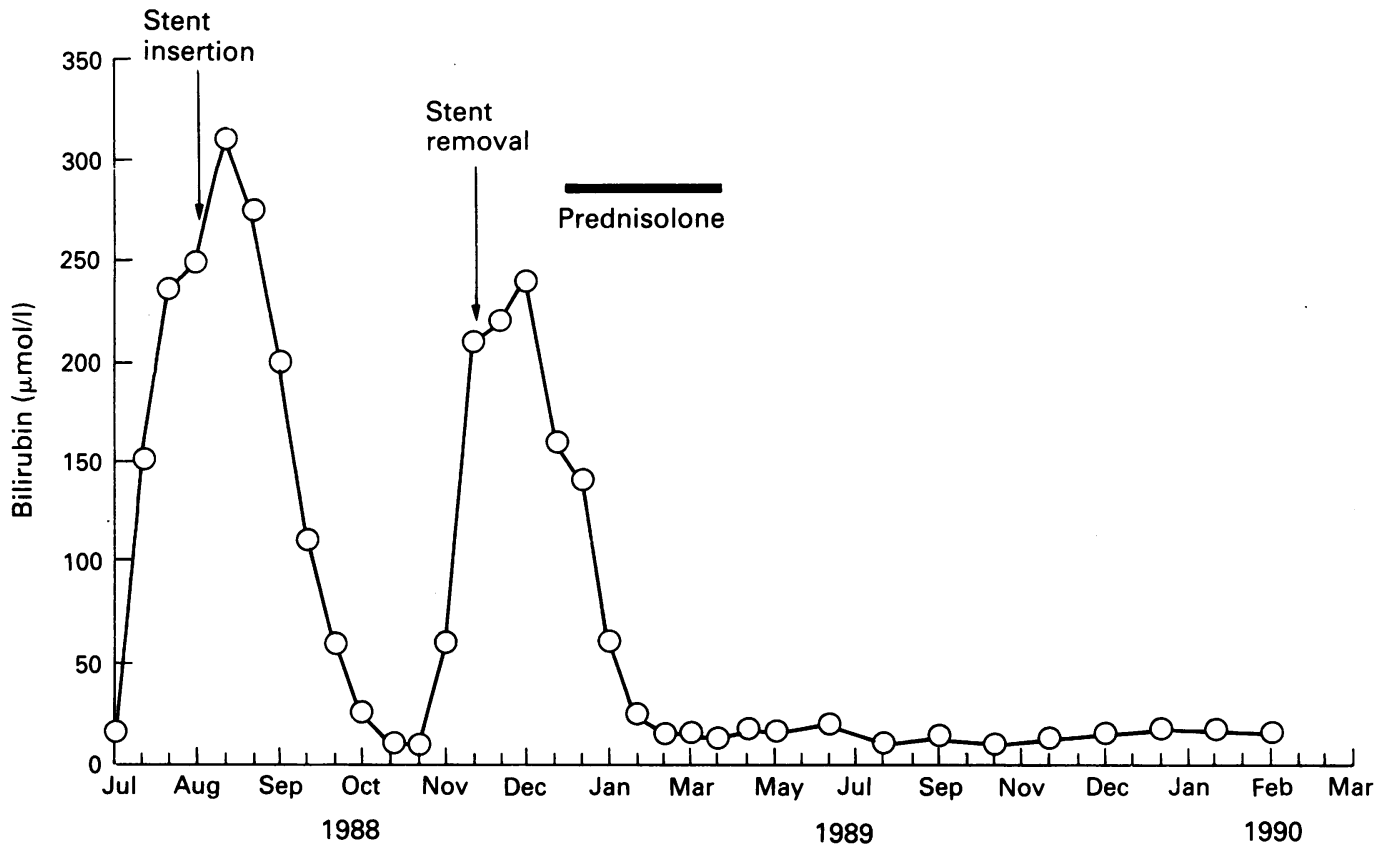

cholestatic nature of her jaundice, the bilirubin concentration having risen to $243 \mu \mathrm{mol} / \mathrm{l}$ (Fig 2). Ultrasonography showed dilated intrahepatic ducts, and a percutaneous transhepatic cholangiogram was performed (Fig 3) and showed multiple strictures within the biliary tree consistent with sclerosing cholangitis. A stent was inserted to allow bile drainage.

Histology of the liver showed features of biliary obstruction with fibrous portal expansion, bile ductular proliferation, cholestasis, and occasional bile infarcts. A few granules of copper-associated protein were deposited in periportal hepatocytes indicating an element of chronic cholestasis. Appearances were consistent with sclerosing cholangitis, although diag-

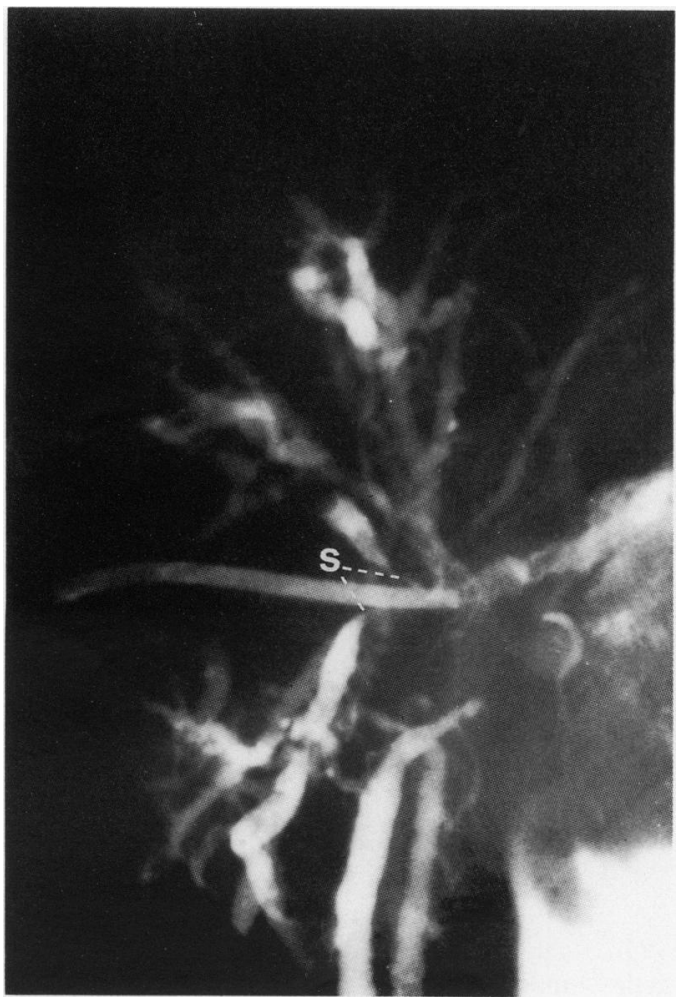

nostic duct lesions were not evident. Abdominal computed tomography suggested a mass in the head of the pancreas extending to the hilum of the liver. A laparoscopic biopsy specimen of this mass (Fig 4) showed diffuse infiltration of hilar connective tissue by inflammatory cells and fibroblasts producing a picture similar to that seen in the original thyroid biopsy specimen. A few bile duct structures were present but there was no evidence of malignancy. Her jaundice improved with stenting and she was allowed home.

In November 1988 she became unwell again with deepening jaundice (Fig 2) caused by blockage of the stent. Endoscopic relief was difficult and because malignancy had not been totally excluded a laparotomy was performed. At operation a large, hard mass surrounding the pancreatic head with extensive infiltration of the hilum of the liver was noted. There was also extensive fibrous thickening of the retroperitoneum. Operative biopsy specimens showed features of chronic pancreatitis with fibrosis and chronic inflammation in the retroperitoneal extrapancreatic area but no evidence of neoplasia. Postoperatively, a normal intravenous urogram was performed and the patient was

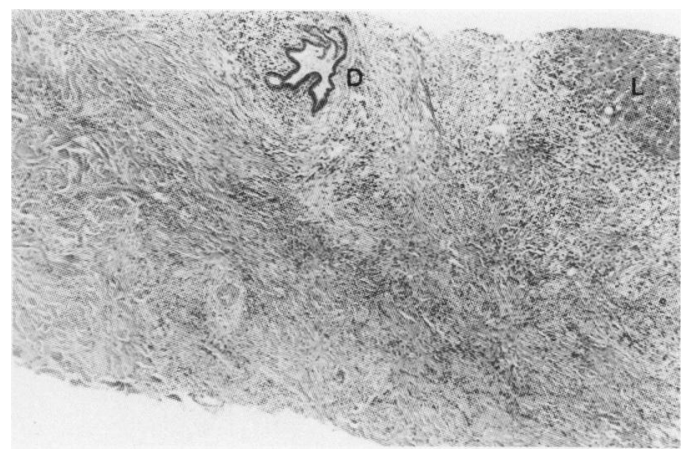

Figure 4: Hilar connective tissue with diffuse infiltration by chronic inflammatory cells. Appearances are non-specific but similar to those seen in the thyroid specimen (Fig 1 ). A small bile duct $(D)$ and normal liver parencyma $(L)$ can be seen. $($ Haematoxylin and eosin original magnification $\times 70$.) 
begun on steroids on an empirical basis (Fig 2). Her bilirubin concentration, which had begun to fall after stent removal, continued to improve and the steroids were slowly reduced to zero over a period of two months.

At recent review two years after the onset of jaundice she remains well with a normal bilirubin concentration and slightly raised liver enzyme activities.

\section{Discussion}

We have described the unusual association of Riedel's thyroiditis, retroperitoneal fibrosis, and sclerosing cholangitis. In this patient the progression of the disease from presentation was rapid suggesting a malignant process. No evidence for this could be found, however, with biopsy specimens showing a similar fibrotic inflammatory process affecting a number of different sites.

Whether the biliary obstruction caused by sclerosing cholangitis was self limiting or brought into remission by steroid treatment is unclear. Initial improvement followed stent insertion and no relapse occurred after stent removal. Steroids were begun soon after this and in the absence of further relapse it is possible that they have had an effect on the disease. The observations of Bartholemew' and Gleeson ${ }^{2}$ support this theory. Their patients had overlap fibrotic syndromes with biliary obstruction caused by sclerosing cholangitis, and in both cases this was relieved by steroid treatment. Other workers ${ }^{89}$ have also found steroids to be of benefit in fibrotic overlap syndromes, and cyclophosphamide has been used with success to suppress fibrosis affecting the thyroid, face and orbit. ${ }^{10}$ In all these cases extrathyroidal fibrosis, in the retroperitoneal space and around the biliary tree, seemed more sensitive to steroid treatment than thyroiditis, which showed an unpredictable response. This response to steroids suggests a different disease process to that which occurs when retroperitoneal fibrosis or primary sclerosing cholangitis are found alone where the results of steroid treatment are unpredictable. ${ }^{\prime \prime \prime}$ ?

The aetiology of these fibrotic conditions is not known but it seems that in overlap conditions immunosuppression is of value in suppressing fibrosis in and around the biliary tree. This suggests that there may be an inappropriate immune response, but the rarity of the condition currently limits further investigation of this hypothesis.

1 Bartholemew LG, Cain JC, Woolner LB, Ut\% DC. Sclerosing cholangitis. Its possible association with Riedel's thyroiditis and fibrous retroperitonitis. Reports of two cases. $N$ Engl and fibrous retroperito

2 Gleeson MH, Taylor S, Dowling RH. Multifocal fibrosclerosis. Proc $R$ Soc Med 1970; 63: 1309-11

3 Mitchinson MJ. Bowlby's disease. BMF 1971; 4: 46-7.

4 Barrett NR. Idiopathic mediastinal fibrosis. B. F Surg 1958; 46: 207.

5 Lillemoe KD, Pitt HA, Cameron JL. Sclerosing cholangitis. Adv Surg 1987; 21: 65-92.

6 Hache L, Woolner LB, Bernat: PE. Idiopathic fibrous mediastinitis. Dis Chest 1962; 41: 9-25.

7 Woolner LB, McConahey WM, Beahrs OH. The surgical aspects of thyroiditis. Am $\mathcal{F}$ Surg 1962; 104: 666-71.

8 McDowell RAW. Cervical and mediastinal fibrosis presenting with thyroid swelling. BMF 1971; 3: 290 .

9 Sjogren I, Wengle B, Korsgren M. Primary sclerosing cholangitis associated with fibrosis of submandibular glands and the pancreas. Acta Med Scand 1979; 205: 139-41.

10 Amorosa LF, Shear K, Spiera H. Multifocal fibrosis involving the thyroid face and orbits. Arch Intern Med 1976; 136 221-3.

11 Koep L, Zuidema GD. The clinical significance of retroperitoneal fibrosis. Surgery 1977; 81: $250-7$.

12 Baker LRI, Mallinson WJW, Gregory MC, Menzies EAD, Cattell WR, Whitfield WN, et al. Idiopathic retroperitoneal fibrosis. A retrospective analysis of 60 cases. $\mathrm{Br} \mathcal{F}$ Urol 1988 ; 60: 497-503. 